\title{
Structural and electrical studies of partial dislocations and stacking faults in (11-20)-oriented $4 \mathrm{H}-\mathrm{SiC}$
}

\author{
L. Ottaviani ${ }^{* 1}$, H. Idrissi ${ }^{1}$, P. Hidalgo ${ }^{2}$, M. Lancin ${ }^{1}$, and B. Pichaud ${ }^{1}$ \\ ${ }^{1}$ TECSEN UMR 6122, Case 231, Paul Cézanne University, Aix-Marseille III, 13397 Marseille Cedex 20, \\ France \\ 2 Departamento de Fisica de Materiales, Faculdad de Ciencias de Fisicas, Universidad Complutense de \\ Madrid, 28040 Madrid, Spain
}

Received 11 September 2004, revised 14 October 2004, accepted 14 October 2004 Published online 7 April 2005

PACS 61.72.Hh, 61.72.Nn, 72.10.Fk, 72.80.Cw, 78.60.Hk

This paper presents cathodoluminescence, electrical and structural characteristics of (11-20)-oriented 4H$\mathrm{SiC}$ substrates, aiming at determining properties of some extended defects. As-grown basal plane dislocations with the Burgers vector $b=1 / 3<11-20>$ previously revealed to be associated to a radiative recombination level at $1.80 \mathrm{eV}$. Well controlled dislocations were here introduced by annealing the sample under compressive stress at $973 \mathrm{~K}$. After the annealing, double stacking faults were detected, formed by two Shockley partial dislocations gliding in two successive basal planes. These defects proved to introduce a rectifying behaviour during forward voltage operation, with a corresponding barrier height value of 0.58 $\mathrm{eV}$ at room temperature. Cathodoluminescence measurements allowed to attribute a radiative level at 1.80 $\mathrm{eV}$ to the extended defects, giving rise to a double luminescence peak.

(C) 2005 WILEY-VCH Verlag GmbH \& Co. KGaA, Weinheim

\section{Introduction}

Silicon Carbide ( $\mathrm{SiC}$ ) is a wide band-gap semiconductor, having opto-electronic properties suitable for many applications, especially high power and high frequency devices. Some structural defects due to crystal growth and/or processing technologies are commonly present in the substrate. It is therefore necessary to investigate the properties of these defects, in order to determine their influence on the optical and electrical properties of the material. In particular, (11-20)-oriented $4 \mathrm{H}-\mathrm{SiC}$ samples are mainly studied today, through two distinct improvements with respect to (0001) Si face : a better channel mobility measured for planar MOSFETS [1], and a better crystal reordering during post-ion implantation annealing [2]. Studies of structural and electrical properties of substrates with this peculiar orientation are then crucial.

Recently, a degradation phenomenon associated with the growth of defects was observed in $4 \mathrm{H}-\mathrm{SiC} \mathrm{p}$ $\mathrm{n}$ diodes during forward-bias reliability testing. The responsible defects were identified as single stacking-faults (SSFs) bound by Shockley partial dislocations [3]. Additionally, the formation of double stacking faults (DSFs) inducing a $4 \mathrm{H} \rightarrow 3 \mathrm{C}$ polytypic transformation was observed to occur in highly nitrogen doped $4 \mathrm{H}-\mathrm{SiC}$ at typical device processing temperatures [4], or during oxidation [5]. The (11-20)-4H SiC orientation is very interesting for controlling and studying these kinds of defects, since their introduction by external mechanical stress is easier due to a higher Schmidt factor : $\mathrm{m}\{[-2110](0001)\}=0.43$, while $\mathrm{m}\{[11-20](0001)\}=0$ for (0001)-orientation. As-grown basal plane dislocations (BPDs) with the Bur-

" Corresponding author: e-mail: Laurent.Ottaviani@univ.u-3mrs.fr, Phone: +33 49128 83 46, Fax: +33 491288852 
gers vector $b=1 / 3<11-20>$ revealed to be associated to a radiative recombination level at $1.80 \mathrm{eV}$ [6]. Well-controlled dislocations were then introduced, in order to be compared with such as-grown defects.

\section{Experiment}

We used (11-20) oriented 4H-SiC nitrogen-doped $\left(5 \times 10^{18} \mathrm{~cm}^{-3}\right)$ wafers provided by Cree Research. The $\{0001\}$ glide planes were perpendicular to the sample surface and at $45^{\circ}$ from [2-201] orientation, which is the tension-compression axis. The samples were deformed by cantilever bending and annealed under stress at $973 \mathrm{~K}$ during $10 \mathrm{mn}$. Dislocations were nucleated from the sample edge damaged by wafer cutting. The defects emerging at the surface were characterized by chemical etching using molten $\mathrm{KOH}$ $\left(500{ }^{\circ} \mathrm{C}, 10 \mathrm{mn}\right)$. (11-20) planar views from HRTEM imaging were prepared by mechanical polishing and ion beam milling of the sample back sides. A 2010-FEG was used at $200 \mathrm{keV}$ (CP2M-Marseille).

Cathodoluminescence (CL) measurements were performed in a Hitachi S-2500 scanning electron microscope, equipped with a computer controller Oriel 7720 monochromator and a Hamamatsu R298 photomultiplier for detection. Details of the experimental set-up for spectral and panchromatic CL measurements are presented elsewhere [7]. Current-voltage measurements were carried out using a Keithley 4200 system.

\section{Results and discussion}

\subsection{Structural observations}

Optical images of deformed and chemically etched sample revealed straight lines parallel to the (0001) basal plane, which corresponds to the intersection of macroscopic SFs with the surface. Partial dislocations were nucleated from the sample edge, and expanded towards [-1100] direction (see Fig. 1). This expansion is rapid, due to the applied compressive stress.

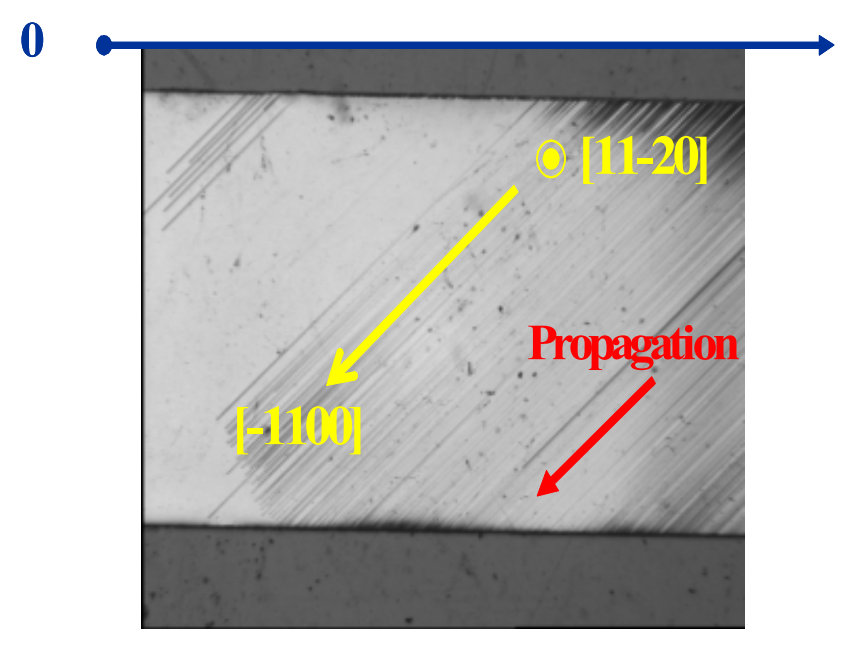

Fig. 1 Optical microscopy of deformed and annealed sample.
HRTEM images showed that each macroscopic line consists of one DSF, bound by a pair of leading $30^{\circ}$ partial $\mathrm{Si}(\mathrm{g})$ dislocations aligned along the [-12-10] direction, and exhibiting the same Burgers vector $b= \pm a / 3[-1100]$ (see Fig. 2). The trailing dislocations are not seen, due to an annealing temperature lower than the critical value for their expansion.

$3 \mathrm{C}$ layers consisting of 6 planes in the $4 \mathrm{H}-\mathrm{SiC}$ matrix are clearly observed : such defects can only be obtained if the two identical partials glide on two adjacent basal planes.

This configuration, giving a varying density of BPDs and DSFs on the sample surface along the [2-201] axis, is very useful for studying their influence on electrical and optical characteristics. 


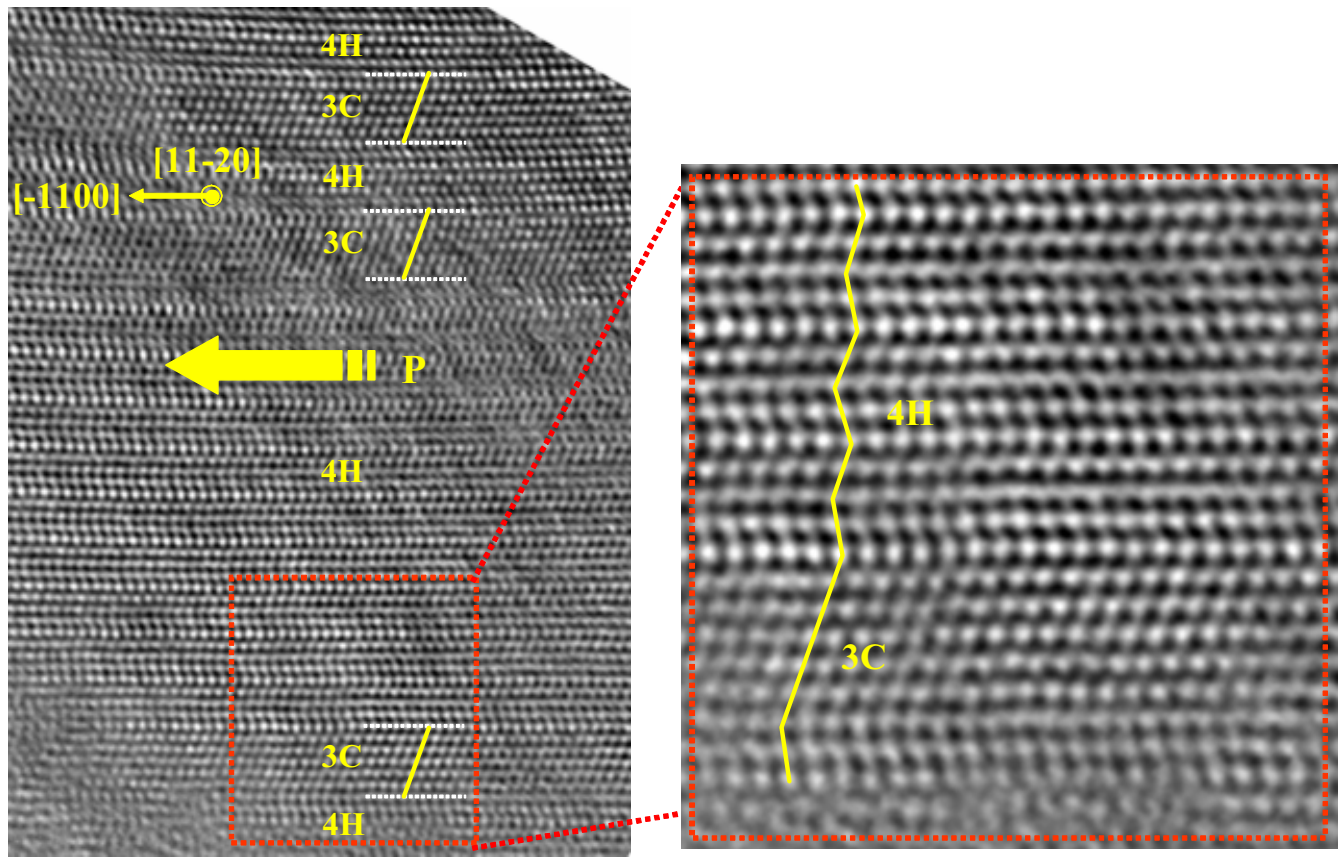

Fig. 2 [11-20] HRTEM image (white atoms) of deformed and annealed sample.

\subsection{Electrical observations}

Hall Effect measurements were first carried out, for studying the influence of BPDs and DSFs on lateral conduction. The I-V curves denoted a loss of ohmicity above the region containing a high density of extended defects [8]. Between the zone without any defect and the highest defect-density zone, the electron mobility decreased from 15.1 to $11.3 \mathrm{~cm}^{2} / \mathrm{V}$.s, and resistivity increased from 1.47 to $1.82 \mathrm{~m} \Omega . \mathrm{cm}$.

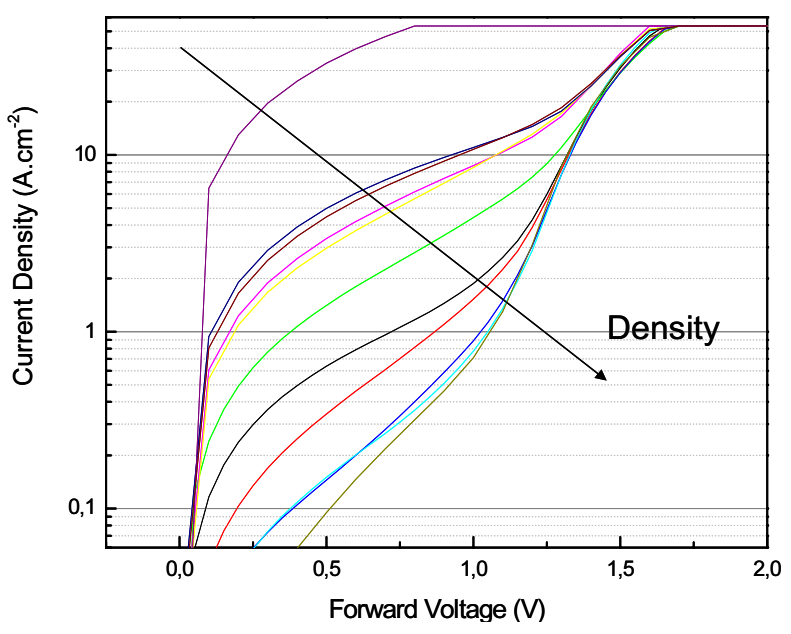

Fig. 3 Forward current-voltage characteristics vs basal plane dislocation density.
Ni Schottky contacts were then patterned (150 $\mathrm{nm}$ thick, $0.5 \mathrm{~mm}$ diameter). Figure 3 compares forward current-voltage characteristics with respect to BPD density.

Extended defects clearly induce a rectifying behaviour visible above $1 \mathrm{~V}$, becoming more important with BPD density. An increase of the transverse resistance is also evident, which was already deduced from Hall Effect measurements.

Figure $4 \mathrm{a}$ shows I-V-T characteristics of a diode patterned on a defect-free region. Despite

the deposited Schottky contact, the diode behaviour is purely ohmic due to the high doping of the substrate. The measured resistance remains constant with temperature, at a value (8.7 $\Omega$ ) superior to the bulk resistance due to the nonideality of the contact. 


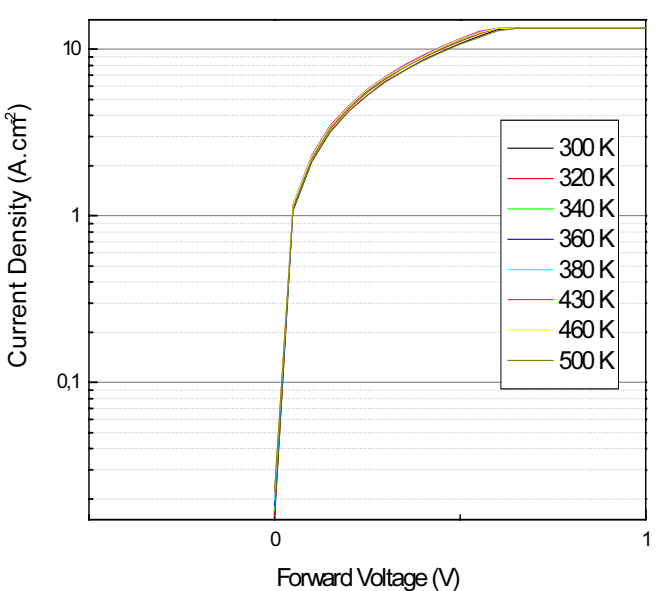

Fig. 4a Current-voltage curves: on defect-free region

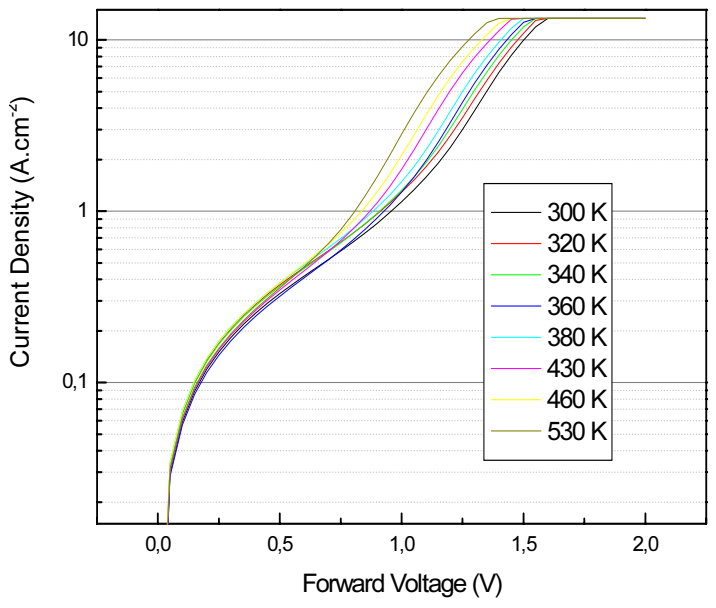

Fig. $4 \mathbf{b}$ on high-BPD density region.

Current-voltage curves (with $\mathrm{T}$ as parameter) extracted from high-BPD density region can be depicted with the very simple scheme given in Fig. 5. The parallel resistance $\mathrm{R}_{\mathrm{p}}$ remains constant (with $\mathrm{T}$ ) at 172 $\Omega$. The rectifying behaviour becomes prominent when a threshold voltage is attained, and a series resistance $\mathrm{R}_{\mathrm{s}}$ is measured for high voltages (before set-up compliance) at $3.1 \Omega$, which is the same order of magnitude than the defect-free region resistance.

The rectifying behaviour could be due to a depletion zone created by quantum wells (QWs) in $4 \mathrm{H}$

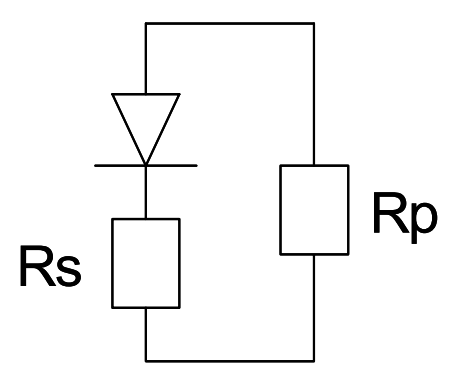

Fig. 5 Defect electrical model. matrix, which have proven to be associated with the $3 \mathrm{C}$ lamellae forming DSFs in highly doped regions [9]. Figure 6 gives the corresponding barrier heights $\Phi(\mathrm{T})$, extracted from the currentvoltage curves with the following relation:

$I_{s}=S A^{*} T^{2} \exp (-q \Phi / k T)$

Is is the saturation current, measured after subtraction of the I$\mathrm{V}$ region depending on $\mathrm{R}_{\mathrm{p}} \cdot \mathrm{A}^{*}$ is the Richardson constant (146 $\mathrm{A} / \mathrm{cm}^{2} \mathrm{~K}^{2}$ ), and $\mathrm{S}$ is the contact surface.

The barrier height calculated at room temperature is $0.58 \mathrm{eV}$. This value seems correct, since the lowest electron energy associated with DSFs was calculated to be about 0.60 $\mathrm{eV}$ below the conduction band [4].

Moreover, a twin exciton signature was recently

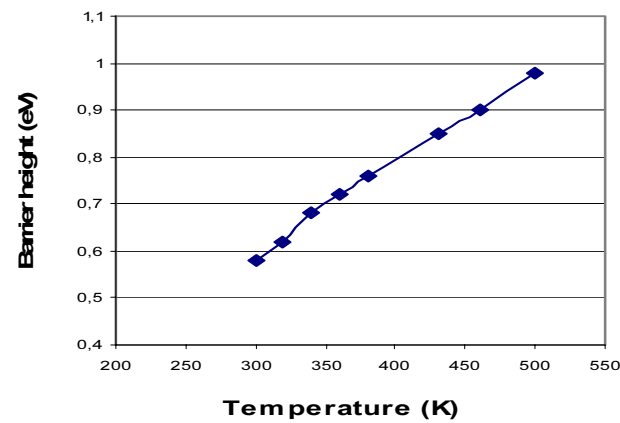
assigned to SFs, allowing two possible electron-hole recombinations [11]. At room temperature, the transition between localised electrons and holes confined at the highest energy interface give rise to a calculated transition energy of $2.5 \mathrm{eV}$ for 7 bilayers. This energy is close to the $4 \mathrm{H}-\mathrm{SiC}$ band gap value diminished by the barrier height we determined.

When temperature rises, the Fermi level approaches the center of the band gap and comes below the fault energy level. Therefore, QW electron density decreases [12] and barrier height increases, thus reducing the influence of the defects on electrical char-

Fig. 6 Barrier height variation with $\mathrm{T}$. acteristic. 
3.3 Cathodoluminescence observations $(20 \mathrm{kV})$

Panchromatic CL images show straight lines corresponding to BPDs, with a varying intensity along the sample edge. Spectra acquired at $77 \mathrm{~K}$ show a clear influence of defect concentration (see Fig. 7, for very low excitation level). For a high BPD density, the green band gets a higher intensity than other classical luminescence peaks, being more important (with extinction of the band gap signature) when excitation level is reduced. In this case, two Gaussian curves can be fitted, and a clear asymmetry is detected around $1.8 \mathrm{eV}$, meaning that two peaks are involved. Emissions in the green band region have lower intensities in defect-free regions. The band gap emission is still present at low excitation, proving a poor concentration of radiative centres associated to green luminescence.

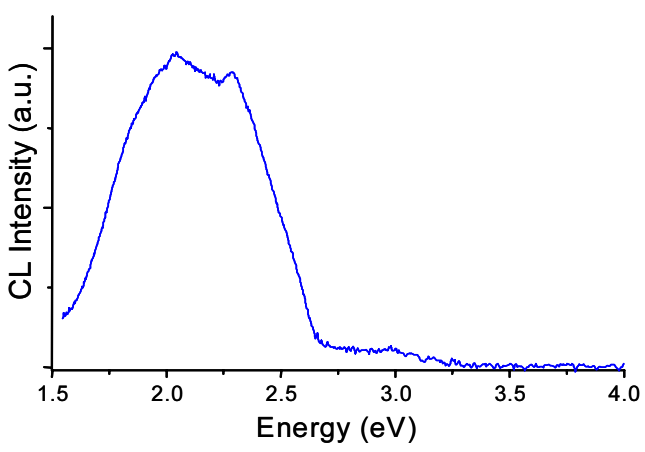

Fig. 7a CL spectra on high BPD density region

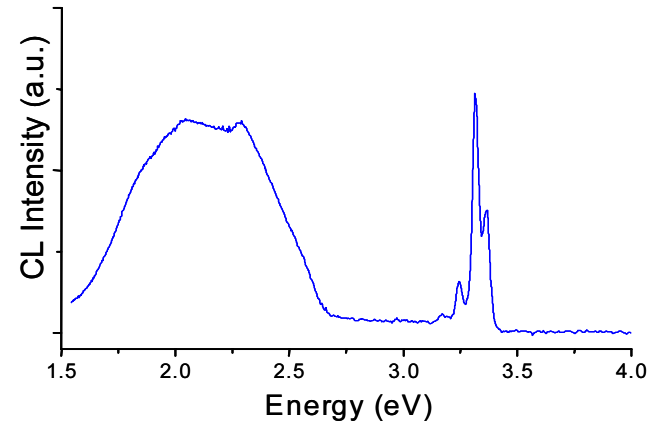

Fig. 7b CL spectra on defect free region.

\section{Summary}

This paper presents structural, electrical and CL characterisations of samples, aiming at determining properties of extended defects introduced by annealing the sample under compressive stress at $973 \mathrm{~K}$. Chemical etchings revealed the presence of double stacking faults formed by two Shockley gliding in two successive basal planes. These faults proved to introduce quantum well structures in the matrix, leading to depletion zones and corresponding barrier heights. CL measurements showed a luminescence at $1.80 \mathrm{eV}$ associated to extended defects, with a double peak not visible in as-grown dislocations.

\section{References}

[1] H. Yano, T. Hirao ; T. Kimoto, H. Matsunami, IEEE Electron Dev. Lett. 20, 611 (1999).

[2] J. Wong-Leung, M. Linnarsson, B. Svensson, Physica B 340, 132 (2003).

[3] J. Liu, M. Skowronski, C. Hallin, R. Soderholm, H. Lendenmann, Appl. Phys. Lett. 80, 749 (2002).

[4] J. Liu, H. Chung, T. Kuhr, Q. Li, M. Skowronski, Appl. Phys. Lett. 80, 2111 (2002).

[5] R. Okojie, M. Xhang, P. Pirouz, S. Tumakha, G. Jessen, L. Brillsson, Appl. Phys. Lett. 79, 3056 (2001).

[6] L. Ottaviani, P. Hidalgo, H. Idrissi, M. Lancin, S. Martinuzzi, B. Pichaud, J. Phys.: Condens. Matter 16, S107 (2004).

[7] P. Hidalgo, B. Méndez, P. Dutta, J. Piqueras, E. Dieguez, Phys. Rev. B 57, 6479 (1998).

[8] M. Zhang, H. Hobgood, M. Treu, P. Pirouz, Mater. Sci. For. 457-460, 759 (2003).

[9] B. Skromme, M. Mikhov, L. Chen, G. Samson, R. Wang, C. Li, I. Bhat, Mater. Sci. Forum 457-460, 581 (2003).

[10] S.M. Sze, Physics of Semiconductor Devices (Wiley-Interscience, New Jersey, 1969), chap. 8.

[11] S. Juillaguet,in: Proceedings of the 5th European Conference on Silicon Carbide and Related Materials, Bologna, Italy, August 31-September 4 2004, p. 240.

[12] T. Kuhr, J. Liu, H. Chung, M. Skowronski, F. Szmulowicz, J. Appl. Phys. 92, 5863 (2002). 